\title{
Multichannel SAR Imaging with Backprojection
}

\author{
\# Luke Rosenberg ${ }^{1-3}$ and Doug Gray ${ }^{1-2}$ \\ ${ }^{1}$ Electrical Engineering Department, University of Adelaide \\ North Terrace, Adelaide SA, Australia 5005 \\ email: luker@eleceng.adelaide.edu.au \\ email:dgray@eleceng.adelaide.edu.au \\ ${ }^{2}$ Cooperative Research Center for Sensor, Signal and Information Processing (CSSIP) \\ ${ }^{3}$ Defence, Science and Technology Organisation (DSTO)
}

\begin{abstract}
The use of multiple antennas on a Synthetic Aperture Radar (SAR), ie. multichannel SAR offers benefits such as improved ambiguity suppression for Moving Target Indication (MTI) and imaging large swaths [1], [2], improved Signal to Noise Ratio (SNR) [3] and the potential to suppress spatial jammers by use of space time adaptive processing [4].

The multichannel Matched Filter (MF) interpolation imaging scheme presented in [5] has shown to offer a good tradeoff between imaging accuracy and computational complexity. However there are a number of potential problems which affect this algorithm in practice. Backprojection offers a solution to these problems and instead offers a direct tradeoff between accuracy and computation time.

This work extends the single channel backprojection algorithm to include multiple transmit and receive antennas. An analysis of the performance of the algorithm with varying levels of accuracy is shown as well as a comparison with conventional MF algorithms.
\end{abstract}

\section{INTRODUCTION}

Previous work in multichannel SAR imaging has covered MF processing [4], orthogonal reference vectors for MTI [2] and sidelobe suppression vectors for long-range, low pulse repetition frequency or non-ideal antenna patterns [2], [6].

MF processing is concerned with obtaining optimal SNR at each pixel and may contain sidelobe leakage from neighbouring pixels. Orthogonal reference vectors are used to prevent sidelobe leakage and are essentially an MTI technique to form an image without contributions from stationary scatterers. Sidelobe suppression vectors act as a compromise between the two, reducing sidelobe leakage by minimising the total output power of the filter while ensuring enough energy is obtained from the desired pixel. For most cases of SAR imaging, the sidelobe leakage between pixels is low enough that MF processing is acceptable.

Traditional MF imaging techniques such as those presented by Soumekh [7], include spatial MF interpolation, range stacking and Time Domain Correlation (TDC). TDC is the simplest and most computationally intensive of these algorithms as it relies on a separate reference vector for each focussed pixel.
The principle of stationary phase can then be used to formulate wavenumber domain $\left(\omega-k_{u}\right)$ algorithms. Range stacking offers a trade-off for accuracy in azimuth versus a speed up by using a Fast Fourier Transform (FFT) in that domain, while spatial MF interpolation uses FFTs in both domains and requires an interpolation to map the measured data into the spatial frequency domain. Multichannel implementations of these algorithms has been presented in [5]. It was concluded that the spatial MF interpolation offered the best tradeoff for quality to computation time.

Unfortunately, the use of FFT based algorithms can present some unwanted problems. Formulation based problems include wrap-around errors at the edges of the imaging area and artifacts from the frequency domain Stolt interpolation spread over the entire image. Real world problems include the need to acquire a full aperture of pulses before processing can begin and its sensitivity to motion errors in the flight path. These result in poor focus for large integration angles and/or large motion errors [8].

Backprojection offers an alternative to the FFT based algorithms without the processing overhead of the TDC algorithm. The algorithm has been presented as convolution backprojection in [9] and can be formulated as a modification to Computer Aided Tomography. Recent work includes faster implementation methods, [8], multiresolution imaging with quadtree backprojection [10] and enhancement using digital spotlight preprocessing [11]. However, there has not yet been a formulation suitable for multichannel SAR.

This paper presents the MF formulation for multichannel TDC and spatial MF interpolation. A new multichannel backprojection algorithm is then described which avoids the unwanted problems previously described. An analysis of the computation to quality trade-off of the algorithm is shown before a performance comparison using both a Point Spread Function (PSF) and a sample image. Throughout this paper, each array element transmits and receives and range processing has been performed prior to imaging. The algorithms are demonstrated for stripmap SAR, but can also be modified for spotlight modes. 


\section{SYSTEM MODEL}

Consider a SAR travelling along the y-axis, imaging a patch in the slant-plane $x \in\left[X_{c}-X_{0}, X_{c}+X_{0}\right], y \in\left[-Y_{0}, Y_{0}\right]$ which is offset from the flight path by range $X_{c}$. An $N$ channel linear antenna array is used in the azimuth direction with halfwavelength spacing as shown in Figure 1.

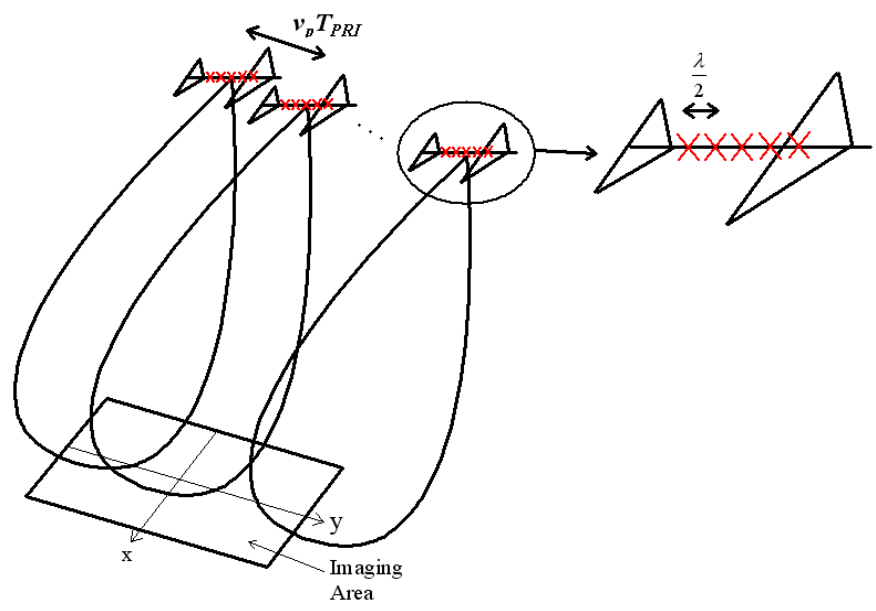

Fig. 1: Multichannel SAR Imaging with $N=5$

After shifting to baseband and range processing, the received signal at the $n^{\text {th }}$ antenna due to a point scatterer at the focus point $(x, y)$ is given by,

$$
s_{n}(t, u, x, y)=\exp \left[-j \omega_{c} \tau_{n}(x, y, u)\right] \operatorname{sinc}\left[B \pi\left(t-\tau_{n}(u, x, y)\right)\right]
$$

where the carrier frequency is $\omega_{c}(\mathrm{rad} / \mathrm{s})$, the transmit bandwidth is $B(\mathrm{~Hz})$ and the variables $(t, u)$ represent fast-time within a pulse and the SAR platform position respectively. The temporal delay is given as twice the distance to the scatterer,

$$
\tau_{\text {temp }, n}(u, x, y)=\frac{2}{c} R(x, y-u)
$$

where $R(\cdot, \cdot)$ is the distance to the patch. The spatial delay is determined by the difference between the $n^{t h}$ and the center reference antenna,

$$
\tau_{\text {spat }, n}(u, x, y)=\frac{1}{c}\left[R\left(x, y-u-d_{n}\right)-R(x, y-u)\right]
$$

with the antenna offset $d_{n}=(n-1) \delta$ for antenna spacing $\delta$ with $n \in[-(N-1) / 2,(N-1) / 2]$ for $N$ (odd) antenna elements. Combined together, the total delay to the $n^{\text {th }}$ channel is given by the sum of the temporal and spatial components,

$$
\begin{aligned}
\tau_{n}(u, x, y) & =\frac{1}{c}\left[R(x, y-u)+R\left(x, y-u-d_{n}\right)\right] \\
& \approx \frac{2}{c} R\left(x, y-u-0.5 d_{n}\right)
\end{aligned}
$$

\section{IMAGING INTRODUCTION}

The imaging system for a single channel can be described by a two dimensional convolution with a transfer function $h(t, u)$

$$
x(t, u)=h(t, u, x, y) \otimes_{t, u} f(x, y)
$$

where $f(x, y)$ is the reflectivity of the patch being imaged. The inverse of this equation can be solved by using a MF of the form $h^{-1}(x, y, t, u)=s^{*}(x, y,-t, u)$.

$$
\begin{aligned}
f(x, y) & =s^{*}(t, u, x, y) \otimes_{t, u} x(t, u) \\
& =\int_{t} \int_{u} s^{*}\left(t^{\prime}-t, u^{\prime}-u, x, y\right) x(t, u) d u d t
\end{aligned}
$$

with $t^{\prime}=\tau(x, y, u)$ and $u^{\prime}=y$. To extend this to multiple channels, the reference and data signals can be stacked for each channel to give the signal vectors

$$
\begin{aligned}
\mathbf{s}(t, u, x, y) & =\left[s_{-(N-1) / 2}(\cdot), \ldots, s_{(N-1) / 2}(\cdot)\right]^{T}, \\
\mathbf{x}(t, u) & =\left[x_{-(N-1) / 2}(\cdot), \ldots, x_{(N-1) / 2}(\cdot)\right]^{T} .
\end{aligned}
$$

and the time-domain imaging equation becomes,

$$
f(x, y)=\int_{t} \int_{u} \mathbf{s}^{H}\left(t^{\prime}-t, u^{\prime}-u, x, y\right) \mathbf{x}(t, u) d u d t
$$

By using Parvesval's theorem, this equation can be represented in the frequency domain. The $(\omega, u)$ domain representation is given as

$$
f(x, y)=\int_{\omega} \int_{u} \mathbf{s}^{H}\left(\omega, u^{\prime}-u, x, y\right) \mathbf{x}(\omega, u) d u d \omega
$$

where the reference vector in the fast-time frequency domain with $\omega \in\left[\omega_{c}-B \pi, \omega_{c}+B \pi\right]$ calculated exactly as,

$$
\begin{aligned}
s_{n}(\omega, u, x, y) & =\exp \left[-j \omega \tau_{n}(u, x, y)\right] \\
& =\exp \left[-2 j k R\left(x, y-u-0.5 d_{n}\right)\right]
\end{aligned}
$$

with the wavenumber $k=\omega / c$. Using the principle of stationary phase, Equation 8 can be written in the $\left(\omega, k_{u}\right)$ domain as

$$
f(x, y)=\int_{\omega} \int_{k_{u}} \mathbf{s}^{H}\left(\omega, k_{u}, x, y\right) \mathbf{x}\left(\omega, k_{u}\right) d k_{u} d \omega
$$

and the slowly fluctuating amplitude terms have not been included. The form of the reference signal in this domain is

$$
s_{n}\left(\omega, k_{u}, x, y\right)=\exp \left[-j \sqrt{4 k^{2}-k_{u}^{2}} x-j k_{u}\left(y-0.5 d_{n}\right)\right]
$$

\section{Time Domain Correlation}

Time Domain Correlation is the most exact SAR imaging algorithm as it solves the imaging Equation 7 precisely for each pixel. It is presented for a single channel by Soumekh [7] and its multichannel equivalent by Rosenberg and Gray, [5]. Its implementation is described here as it is used as a benchmark for the analysis in this paper.

The target function $f(x, y)$ is derived by convolving the SAR signal with a shift-varying filter in the time domain with a reference vector at a given point $(x, y)$. This implies that the reference vector in either the $(t, u)$ or $(\omega, u)$ domain must be calculated with a delay determined for each point. The integration is then performed over both variables to obtain the return for that point. This formulation results in a high computational expense, but also provides highly accurate results. Figure 2 represents this algorithm in a block diagram. 


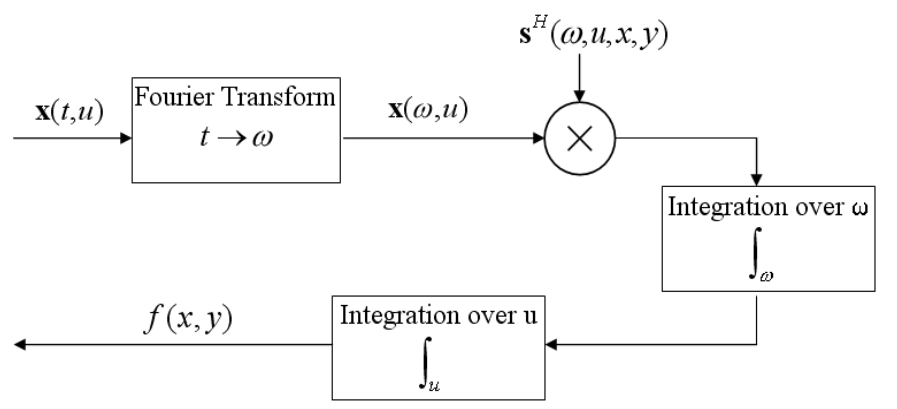

Fig. 2: Time Domain Correlation Block Diagram

\section{SPATIAL MF INTERPOLATION}

Soumkeh [7], has presented this algorithm as spatial frequency interpolation for a single channel SAR. It has also been proposed for multichannel SAR by Ender [4], though his implementation is in the $(\omega, u)$ domain so it can be extended to STAP. He has also shown how this can be derived in the $\left(\omega, k_{u}\right)$ domain for a single range. It is this $\left(\omega, k_{u}\right)$ domain approach using spatial only vectors combined with the multiple range interpolation of [7] that is presented below.

The imaging equation of Equation 9 describes the reference and measurement signals in Fourier Transformed $\left(\omega, k_{u}\right)$ domain. However, we are interested in the image $(x, y)$ domain and need to transform the problem accordingly. To form an estimate of the target reflectivity function, $f(x, y)$, range migration compensation is required and is implemented using a using Stolt Interpolation with a sinc function smoothed with a hamming window in the spatial frequency $\left(k_{x}, k_{y}\right)$ domain If we now make a transformation into the $\left(k_{x}, k_{y}\right)$ domain,

$$
\begin{aligned}
& k_{x}=\sqrt{4 k^{2}-k_{u}^{2}}, \\
& k_{y}=k_{u}
\end{aligned}
$$

then Equation 9 becomes

$$
\begin{array}{r}
f(x, y)=\int_{k_{x}} \int_{k_{y}} \exp \left[j k_{x} x+j k_{y} y\right] \\
x\left(k_{x}, k_{y}\right) J\left(k_{x}, k_{y}\right) d k_{y} d k_{x}
\end{array}
$$

where the Jacobian is approximately constant and does not affect the normalised image results.

The spatial MF solves the imaging Equation 9 by varying $\omega$ and $k_{u}$ with a fixed focus position $\left(X_{c}, 0\right)$. To implement this algorithm for multichannel SAR, a reference vector is chosen, $\mathbf{s}_{\text {ref }}\left(\omega, k_{u}\right)=\mathbf{s}\left(\omega, k_{u}, X_{c}, 0\right)$ and hence the image in the $\left(\omega, k_{u}\right)$ domain can be determined by the following inner product,

$$
F\left(\omega, k_{u}\right)=\mathbf{s}_{\text {ref }}^{H}\left(\omega, k_{u}\right) \mathbf{x}\left(\omega, k_{u}\right)
$$

Transformation using the Stolt interpolation is then required to obtain the spatial domain representation in the $\left(k_{x}, k_{y}\right)$ domain, followed by a two-dimensional inverse FFT to achieve the correct imaging relationship as in Equation 11 with the Jacobian normalised out.

$$
f(x, y)=\int_{k_{x}} \int_{k_{y}} \exp \left[j k_{x} x+j k_{y} y\right] F\left(k_{x}, k_{y}\right) d k_{y} d k_{x}
$$

A block diagram summarising this algorithm is presented in Figure 3.

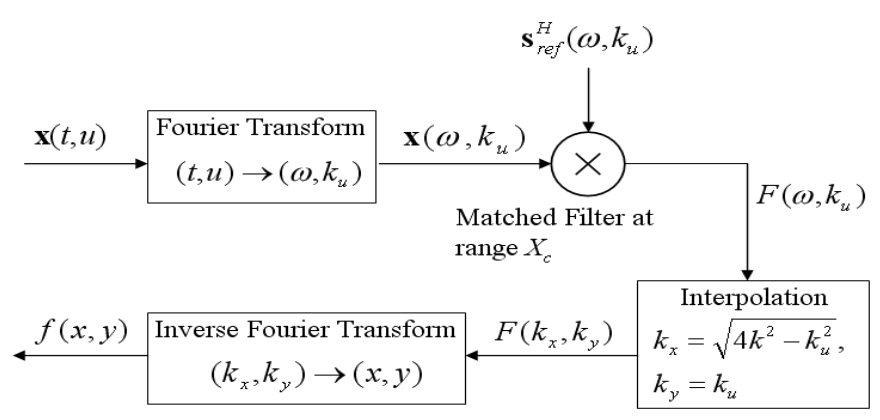

Fig. 3: Spatial Matched Filter Interpolation Block Diagram

\section{BACKPROJECTION}

The third algorithm is multichannel backprojection and offers an alternative time-domain formulation that can be sped up depending on the desired accuracy. For a given SAR location $u$, the $(t, u)$ domain data is traced back (backprojected) to obtain that component of the return for each point $(x, y)$. However, this value also contains contributions from other points at the same slant range. When the delayed returns from a number of SAR locations are integrated, contributions from the point $(x, y)$ add coherently, while returns from other points add incoherently. The backprojection involves interpolating from the measured data to obtain the return for each point. Consider the measured signal from a single channel sampled at each pixel $(x, y)$,

$$
x(t, u) \underset{\text { interp }}{\longrightarrow} x(\tau(x, y, u), u)
$$

where $\tau(x, y, u)=2 \sqrt{x^{2}+(y-u)^{2}} / c$. The time domain interpolation is implemented by Fourier Transforming the data, zero-padding in the $(w, u)$ domain and then Inverse Fourier Transforming. The amount of zero-padding is a tradeoff between the accuracy of the interpolation and the extra computational load. If there are $L$ fast-time samples, then the total number of zero-padded samples is given by $\tilde{L}=Z_{\text {rat }} L$ with the total number of zeros determined by $Z=L\left(Z_{\text {rat }}-1\right)$. A ratio of at least $Z_{\text {rat }}=100$ is typically required for good reconstruction, [7].

If the fast-time slice at SAR location $u$ is defined as $\mathbf{x}(:, u)$, then the baseband interpolated signal is given by

$$
\tilde{\mathbf{x}}_{\mathbf{b}}(:, u)=\mathcal{F}^{-1}\left\{\left[\begin{array}{lll}
\mathbf{0}_{Z / 2} & \mathcal{F}\{\mathbf{x}(:, u)\} & \mathbf{0}_{Z / 2}
\end{array}\right]\right\} \in \mathcal{C}^{\tilde{L} \times 1}
$$

However, for the interpolation to work, the measured data must reverse the baseband conversion performed by the receiver hardware. This is in preparation for the interpolation stage which requires the upsampled data to have a similar phase to the original received data. It must be implemented with the upsampled time $\tilde{t}$, to bring the SAR signal back to the bandpass fast-time frequency, $\omega=\omega_{c}$.

$$
\tilde{x}(\tilde{t}, u)=\tilde{x}_{b}(\tilde{t}, u) \exp \left[j \omega_{c} \tilde{t}\right]
$$




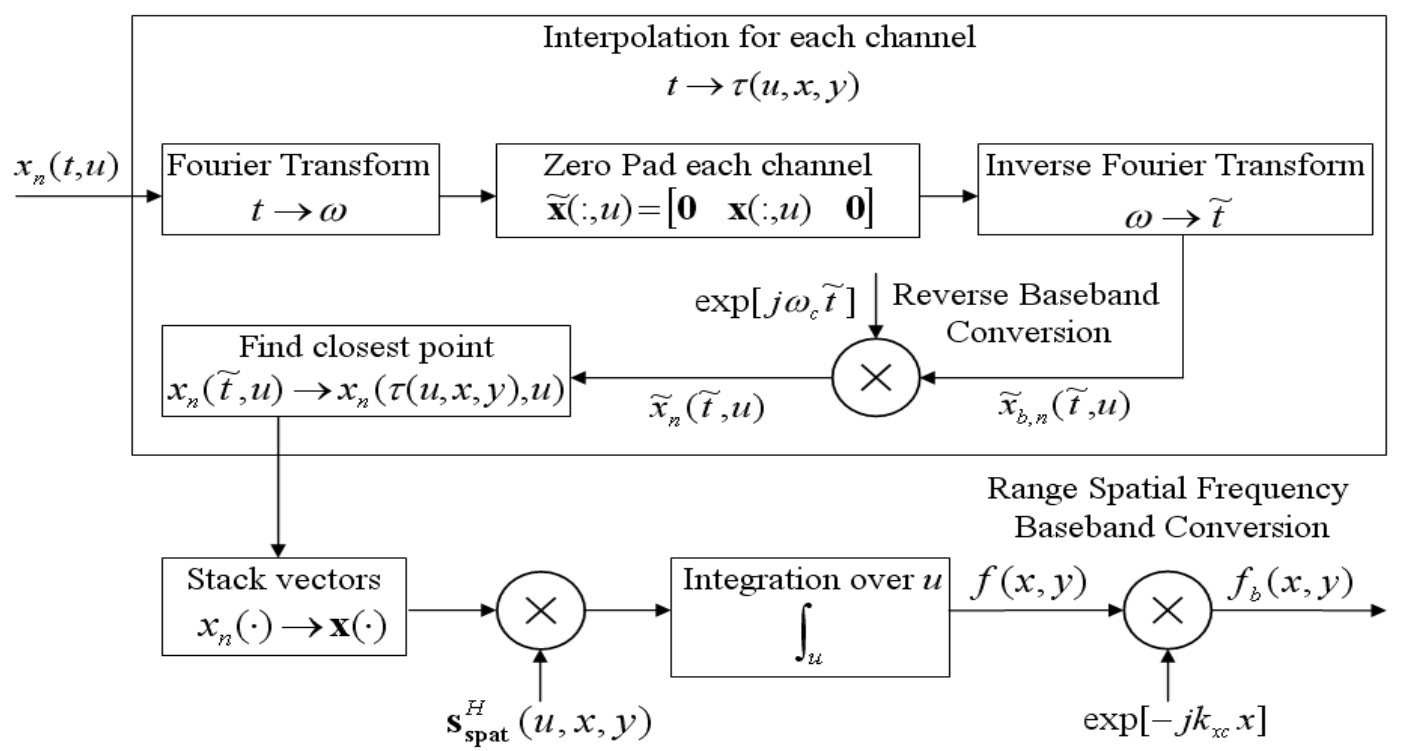

Fig. 4: Backprojection Block Diagram

The interpolation step then finds the closest upsampled point in $\tilde{t}$ to $\tau(x, y, u)$.

$$
\tilde{x}(\tilde{t}, u) \rightarrow x(\tau(x, y, u), u)
$$

The target function can then be determined by integrating over all the pulses.

$$
f(x, y)=\int_{u} x(\tau(x, y, u), u) d u
$$

If phase is important for further analysis, the signal must be coherently basebanded to bring the two-dimensional spectrum to the baseband $\left(k_{x}, k_{y}\right)$ domain.

$$
f_{b}(x, y)=f(x, y) \exp \left[-j k_{x c} x\right]
$$

where $k_{x c}=\left(k_{x, \min }+k_{x, \max }\right) / 2$ and the range spatial frequency $k_{x}$ is given in Equation 10 .

The multichannel extension for this algorithm involves compensating for the spatial phase delay at each interpolated point. It is formed with the delay from Equation 2 and the sinc component approximated to unity.

$$
s_{\text {spat }, n}(u, x, y)=\exp \left[-j \omega_{c} \tau_{\text {spat }, n}(u, x, y)\right]
$$

Once the interpolation has occurred for each channel, both the data vector and the spatial steering vector are stacked as in Equation 6. Equation 17 then becomes,

$$
f(x, y)=\int_{u} \mathbf{s}_{\text {spat }}^{H}(u, x, y) \mathbf{x}(\tau(x, y, u), u) d u
$$

As this algorithm is dependent on the chosen quality of the interpolator, it generally performs better then the $\left(k_{x}, k_{y}\right)$ interpolation, [7]. Figure 4 presents this algorithm in a block diagram.

\section{Simulated Results}

To analyse and compare the backprojection algorithm, an Xband simulation is used with five spatial channels $(N=5)$ arranged in a linear array with half-wavelength spacing. To use the different comparison metrics described in the following section, both a single scatterer at the center of the imaging patch (used to measure the Point Spread Function (PSF)) and a sample image are required for the simulation. The parameters for these scenarios are described in Table 1.

Table 1: Simulation Parameters

\begin{tabular}{|l|l|}
\hline Parameters & Value \\
\hline Carrier Frequency / Bandwidth & $10 / 0.3 \mathrm{GHz}$ \\
Number of Elements / Spacing & $5 / \frac{\lambda}{2} \mathrm{~m}$ \\
Range Resolution / Range Center & $1 \mathrm{~m} / 10 \mathrm{~km}$ \\
Azimuth Resolution & $5 \mathrm{~m}$ \\
PRF / Platform Velocity & $667 \mathrm{~Hz} / 200 \mathrm{~ms}^{-1}$ \\
\hline SAR Imaging Area - PSF & $30 / 6 \mathrm{~m}$ \\
Azimuth / Range Swath Size & $200 / 126$ \\
Number of Pulses / Range Bins & $50 / 7 \mathrm{~m}$ \\
\hline SAR Imaging Area - Sample Image & $200 / 130$ \\
Azimuth / Range Swath Size & Number of Pulses / Range Bins
\end{tabular}

\section{A. Backprojection Performance - PSF}

For an accurate comparison with the other algorithms, it is important to understand how the upsample ratio affects the image quality. Both the PSF and sample image are compared for ratios levels varying between 20 and 150. Figure 5 shows the PSF slices for range and azimuth when the upsampling ratio is set to 20, 50 and 150 respectively.

From this figure, the upsample ratio of 20 appears very distorted, while the ratios of 50 and 150 look very similar. The main difference between the higher ratios are the nulls between sidelobes which are slightly lower for the 150 ratio. A more analytic way of measuring the image quality is to measure the 


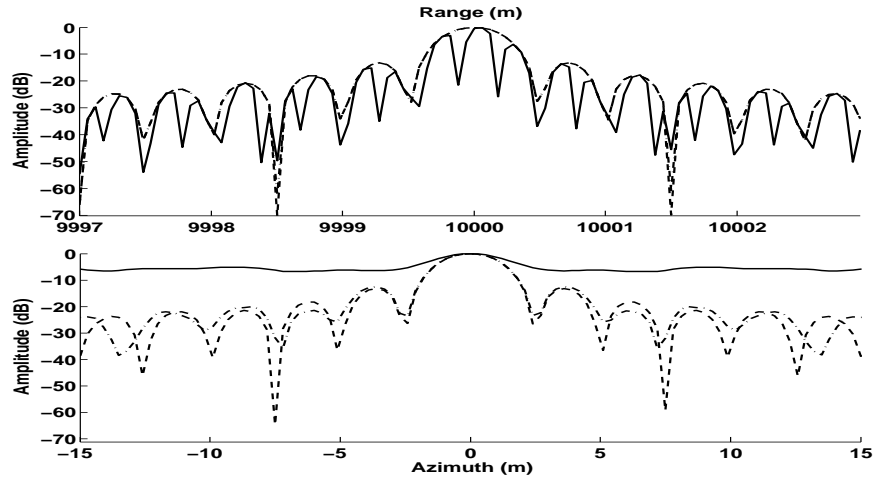

Fig. 5: Backprojection PSF for $Z_{\text {rat }}=20$ (-), 80 (-.-), 150 (- -)

Integrated Sidelobe Ratio (ISLR), which determines the ratio of all energy in the sidelobes to the energy in the mainlobe. Figure 6 shows a comparison of the ISLR for range and azimuth slices of the PSF over the upsampling range.

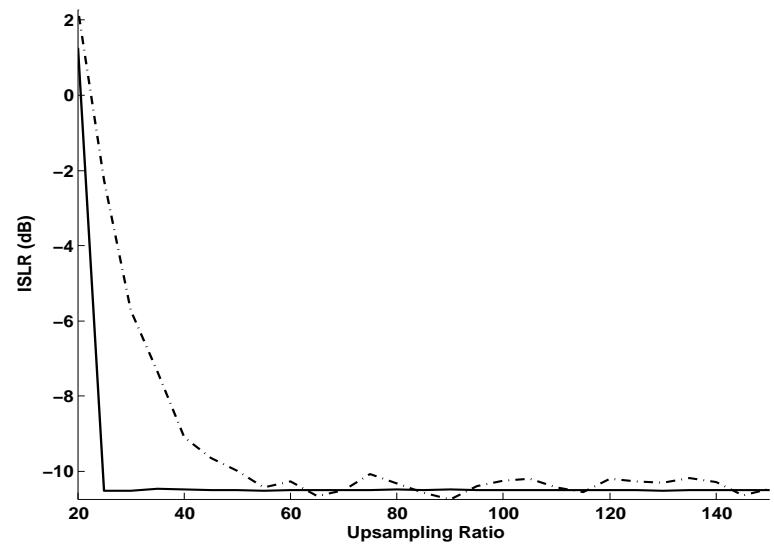

Fig. 6: Integrated Sidelobe Ratio: Range slice (-), Azimuth slice (-.-)

The TDC ISLR's are $-10.49 d B$ for range and $-10.53 d B$ for azimuth. Compared to these results, an upsample ratio of approximately 60 is required to give a reasonable ISLR in both range and azimuth. As the upsample ratio is increased beyond this amount, there is a slight variation due to the varying amounts of sidelobe leakage.

\section{B. Backprojection Performance - Sample Image}

The next results are based on a sample image of the letter ' $S$ '. Figure 7 shows the image before and after imaging with the TDC algorithm.
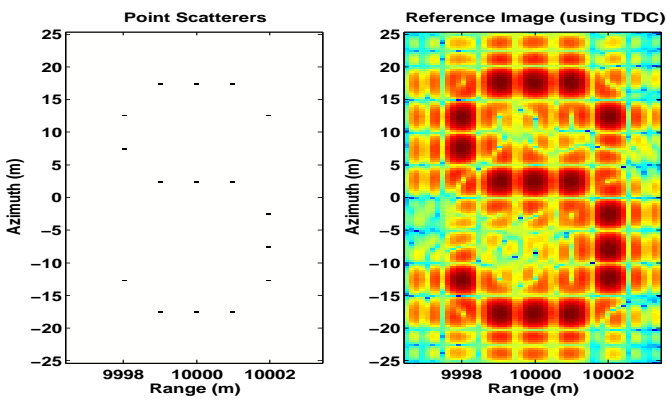

Fig. 7: Sample Image
To visualise the effect of the changing the upsampling ratio, Figure 8 shows the backprojection results as the upsampling ratio is set 20,50 and 150 respectively.

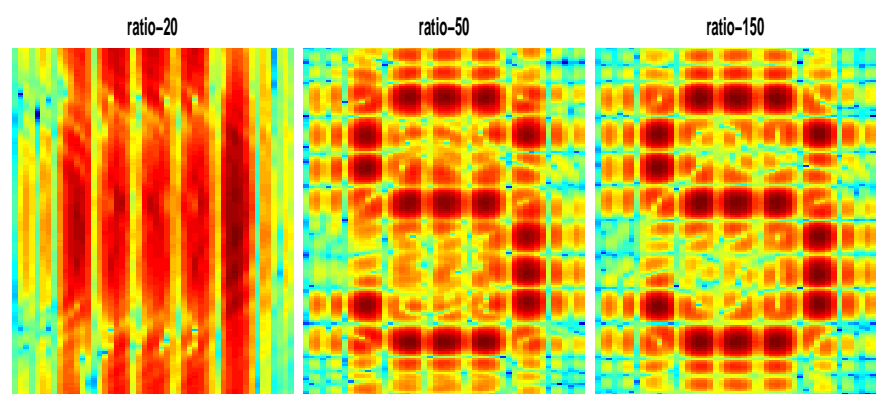

Fig. 8: Algorithm Comparison

Again, it is clear that the ratio of 20 is insufficient to recover an image, while the ratios of 50 and and 150 produce very similar results. To obtain a quantitative measure of image degradation, it is useful to use the TDC result as an ideal reference image and view the backprojection results as a distorted version of the reference image. To this end, the Signal to Distortion Ratio (SDR) and Mean Square Error (MSE) can be calculated. Let $Y\left(x_{p}, y_{q}\right)$ denote the backprojection images for pixels $p=1 \ldots P, q=1 \ldots Q$. Correspondingly, let $D\left(x_{p}, y_{q}\right)$ denote the TDC image. The SDR is then defined as

$$
S D R=\frac{\sum_{p, q}\left|D\left(x_{p}, y_{q}\right)\right|^{2}}{\sum_{p, q}\left|Y\left(x_{p}, y_{q}\right)-D\left(x_{p}, y_{q}\right)\right|^{2}}
$$

Similarly, the MSE is determined by

$$
M S E=\frac{1}{P Q} \sum_{p, q}\left|Y\left(x_{p}, y_{q}\right)-D\left(x_{p}, y_{q}\right)\right|^{2} .
$$

Figure 9 shows a comparison of the SDR and MSE as the upsampling ratio is increased.
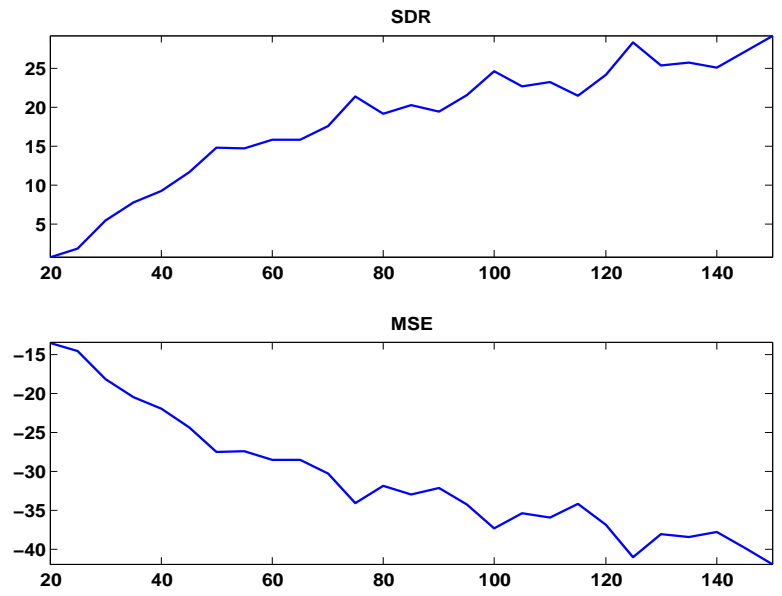

Fig. 9: Backprojection ratio comparison $Z_{\text {rat }}=20(-), 80(-.-), 150$ (-)

From these results, it is clear that overall the SDR increases and the MSE decreases as the upsampling ratio improves. However, there are bumps at each multiple of 25 due to the 
sampling falling into the nulls of the sinc function. In other words, the spectral leakage is at a minimum at these points and there is a slight improvement in the image quality. Soumekh [7], states that a ratio of at least 100 is required for a good quality image and the results in this section agree with that. Use of a higher ratio will still improve the results but only to offer a marginal improvement in quality. It will also slightly increase the run time due to the algorithm complexity (see Table 2).

\section{Algorithm Comparison}

To compare the three algorithms presented in this paper, a simulation has been run with two point scatterers, one in the center of the imaging patch and the other in the top right corner. For the backprojection algorithm, the upsampling ratio for the backprojection has been set to 100 based on the previous results. Simulation results are shown in Figures 10 and 11.

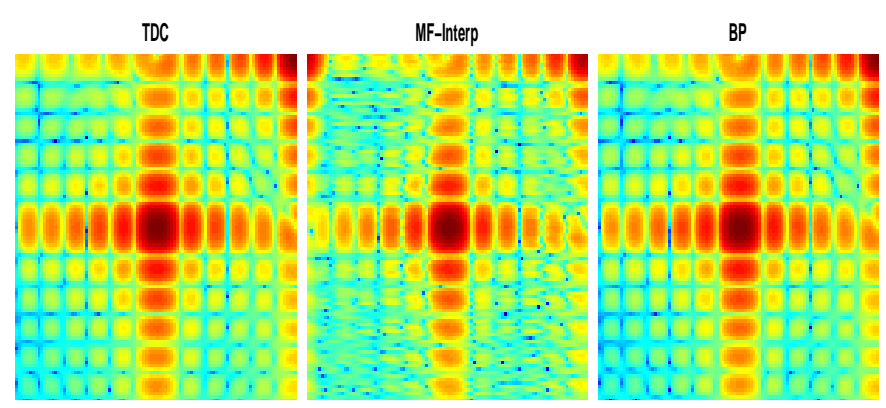

Fig. 10: Algorithm Comparison

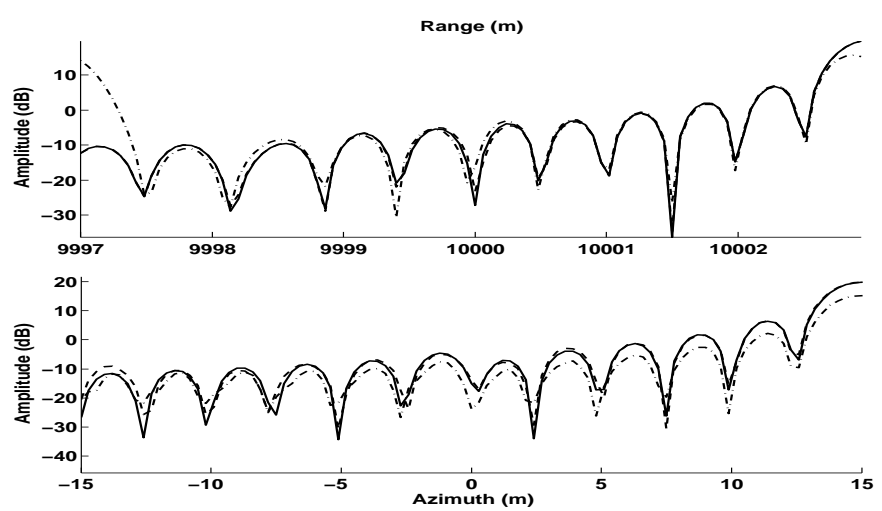

Fig. 11: Algorithm Slice Comparison: TDC (-), MF Interp (-.-), BP (- -)

It can be seen that the spatial MF interpolation algorithm contains aliasing in the range dimension. Further investigation by looking closely at the range slice shows that there is a slight loss in magnitude as well. The time domain correlation and backprojection algorithms look very similar and neither suffer from aliasing or the degradation in magnitude.

The complexity of the three algorithms are shown in Table 2 , where the number of range and azimuth pixels used for the backprojection algorithm is defined as $\left(N_{x}, N_{y}\right)$.
TABLE 2: AlgorithM COMPLEXITY

\begin{tabular}{|l|l|l|}
\hline TDC & Spat. MF Interp. & BP \\
\hline$L^{2} M^{2} N$ & $L M N$ & $\left(N_{x} N_{y}+\tilde{L} \log _{2}(\tilde{L})\right) M N$ \\
\hline
\end{tabular}

The longest time for the backprojection algorithm will be due either to the upsampling ratio or the number of pixels desired for the image.

To get a feel for these results, some realistic parameters for a multichannel SAR may be $N=5$ channels, $M=4246$ pulses and $L=4096$ range bins. Forming an image may require $N_{x}=4096$ range and $N_{y}=4096$ azimuth pixels with an upsample rate of $Z_{\text {rat }}=100$ chosen for backprojection with $\tilde{L}=Z_{\text {rat }} L$. Table 3 shows the complexity calculated with these parameters.

TABle 3: Algorithm Complexity - Real Parameters

\begin{tabular}{|l|l|l|}
\hline TDC & Spat. MF Interp. & BP \\
\hline $1.5 \times 10^{15}$ & $8.7 \times 10^{7}$ & $5.2 \times 10^{11}$ \\
\hline
\end{tabular}

\section{Conclusion}

This paper has demonstrated how the backprojection algorithm can be extended to cater for SAR with multiple receive and transmit antennas. The new algorithm offers a trade-off for quality to computation time and it has been shown that a relatively low level of upsampling is required to approach the image quality of the TDC algorithm. It has also been demonstrated that this algorithm avoids the unwanted aliasing problems associated with spatial MF Interpolation.

\section{REFERENCES}

[1] N.A. Goodman, Sih-Chung-Lin, D. Rajakrishna, and J.M. Stiles. Processing of multiple-receiver spaceborne arrays for wide-area SAR. In IEEE transactions on Geoscience and Remote Sensing, IGARSS, volume 40, no. 4, pages $841-852,2002$.

[2] J.H.G. Ender. Linear and non-linear techniques for multi-channel SAR image generation. In EUSAR 2000, 2000.

[3] M. Younis, C. Fischer, and W. Wiesbeck. Digital beamforming in SAR systems. In IEEE transactions on Geoscience and Remote Sensing Symposium, IGARSS, volume 41, no. 7, pages 1735 -1739, 2003.

[4] J. H. G. Ender. Space-Time Adaptive Processing for Synthetic Aperture Radar. In IEE Colloquium on Space-Time Adaptive Processing, 1998.

[5] L. Rosenberg and D. Gray. Multichannel SAR Imaging using Wavefront Reconstruction. In International Radar Symposium Proceedings, 2004.

[6] N. Goodman, D. Rajakrishna, and J. Stiles. Wide swath, high resolution SAR using multiple receive apertures. In IEEE transactions on Geoscience and Remote Sensing Symposium, IGARSS, volume 3, pages $1767-1769,1999$.

[7] M. Soumekh. Synthetic Aperture Radar Signal Processing with MATLAB Algorithms. John Wiley \& Sons, Inc., 1999.

[8] A.F. Yegulap. Fast Backprojection Algorithm for Synthetic Aperture Radar. In IEEE Radar Conference, 1999.

[9] M.D. Desai and W. Kenneth Jenkins. Convolution Backprojection Image Reconstruction for Spotlight Mode Synthetic Aperture Radar. In IEEE Transactions on Image Processing, volume 1, no. 4, 1992.

[10] Seung mok Oh and J.H. McClellan. Multiresolution imaging with quadtree backprojection. In Asilomar Conference on Signals, Systems and Computers, volume 1, 2001.

[11] L. Nguyen, M. Ressler, D. Wong, and M. Soumekh. Enhancement of backprojection SAR imagery using digital spotlighting preprocessing. In IEEE Radar Conference, 2004. 